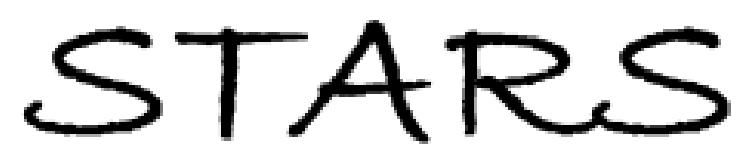

University of Central Florida

STARS

$1-1-2012$

\title{
Anderson localization and colocalization of spatially entangled photons
}

Ayman F. Abouraddy

University of Central Florida

Giovanni Di Giuseppe

University of Central Florida

Demetrios N. Christodoulides

University of Central Florida

Bahaa E. A. Saleh

University of Central Florida

Find similar works at: https://stars.library.ucf.edu/facultybib2010

University of Central Florida Libraries http://library.ucf.edu

This Article is brought to you for free and open access by the Faculty Bibliography at STARS. It has been accepted for inclusion in Faculty Bibliography 2010 s by an authorized administrator of STARS. For more information, please contactSTARS@ucf.edu.

\section{Recommended Citation}

Abouraddy, Ayman F.; Giuseppe, Giovanni Di; Christodoulides, Demetrios N.; and Saleh, Bahaa E. A., "Anderson localization and colocalization of spatially entangled photons" (2012). Faculty Bibliography 2010s. 2185.

https://stars.library.ucf.edu/facultybib2010/2185

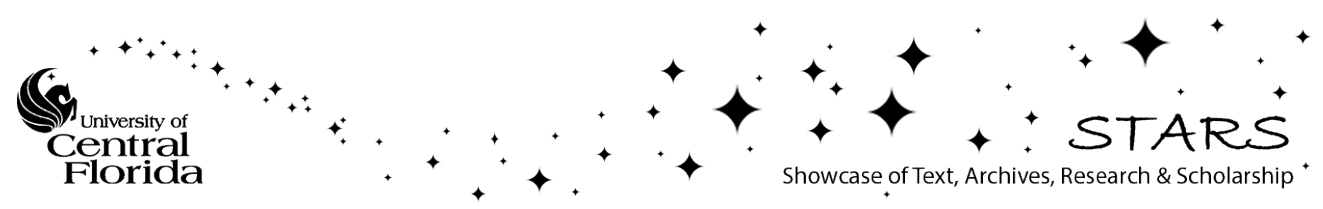




\title{
Anderson localization and colocalization of spatially entangled photons
}

\author{
Ayman F. Abouraddy, ${ }^{1, *}$ Giovanni Di Giuseppe, ${ }^{1,2}$ Demetrios N. Christodoulides, ${ }^{1}$ and Bahaa E. A. Saleh ${ }^{1}$ \\ ${ }^{1}$ CREOL, College of Optics and Photonics, University of Central Florida, Orlando, Florida 32816, USA \\ ${ }^{2}$ School of Science and Technology, Physics Division, University of Camerino, 62032 Camerino, Italy
}

(Received 1 July 2011; published 10 October 2012)

\begin{abstract}
We explore the propagation of light in a two-photon state in disordered optical systems that induce Anderson localization. We show that entangled-photon pairs demonstrate a surprising behavior that we call Anderson colocalization: While neither photon exhibits Anderson localization, the spatial correlations of the pair remain intact. Furthermore, we show that entangled-photon pairs colocalize faster than classical light localizes in the same system. We also explore the propagation of anticorrelated and partially entangled photon pairs in such systems. The results are developed using a linear systems theory that extends the scope of quantum imaging to incorporate disordered systems.
\end{abstract}

DOI: $10.1103 /$ PhysRevA.86.040302

PACS number(s): 03.67.Bg, 42.65.Lm, 72.15.Rn, 42.25.Dd

Quantum mechanical propagation in a disordered lattice may result in a localized wave function, a phenomenon termed Anderson localization (AL) [1]. Observing this fundamental effect, nevertheless, has proven challenging in electronic structures. The analogy between the evolution of a wave function and that of a classical optical field [2] has recently spawned optical realizations of so-called transverse AL [3] in disordered arrays of coupled optical waveguides [4-6]. The exquisite control achieved in state-of-the-art microfabrication [7] now enables studying the propagation of quantum states of light in new classes of photonic circuits [8,9]. Although quantum entanglement is an essential resource in quantum information processing [10], the propagation of spatially entangled photon pairs [11] in an AL system has not been studied extensively heretofore. Since an extended classical source may mask the $\mathrm{AL}$ signature, optical demonstrations of AL using classical light typically make use of point excitations [4]. It might hence be expected that a pair of photons in a spatially entangled state, which is necessarily spatially extended [11], would not exhibit AL upon traversing a disordered lattice. Previous work includes studying two-photon speckle correlations from scattering media [12], examining the impact of disorder on temporal entanglement [13], and the effect of disorder in waveguide arrays on squeezed light [14].

In this Rapid Communication we predict a phenomenon that we call Anderson colocalization (AcL) that may be unraveled in two-photon correlation space. In this phenomenon, each spatially extended photon from an entangled pair remains spatially extended while the relative separation between the two photons in correlation space is localized. We find that entangled photons colocalize faster than classical light localizes in the same system. Furthermore, we investigate the propagation dynamics of entangled photons that are spatially anticorrelated and also the effect of partial entanglement. We uncover a family of two-photon states that are invariant upon passage through an AL system, which we call two-photon AL eigenstates. We present these results using a linear systems theory that extends the formalism of quantum imaging $[11,15]$ to incorporate disordered systems.

*raddy@creol.ucf.edu
We first consider the propagation of a classical optical field in a linear disordered system. A single realization of the scalar input $E_{\mathrm{i}}$ and output $E_{\mathrm{o}}$ fields are related by $E_{\mathrm{o}}\left(x_{1}\right)=$ $\int d x h_{\xi}\left(x_{1}, x\right) E_{\mathrm{i}}(x)$, where $\xi$ refers to the random parameters characterizing the system. The input and output second-order correlation functions [16] are thus related through

$$
G_{\mathrm{o}}^{(1)}\left(x_{1}, x_{2}\right)=\iint d x d x^{\prime} H\left(x_{1}, x_{2} ; x, x^{\prime}\right) G_{\mathrm{i}}^{(1)}\left(x, x^{\prime}\right),
$$

where $x_{1}$ and $x_{2}\left(x\right.$ and $\left.x^{\prime}\right)$ are points in the output (input) plane, $G_{\mathrm{i}}^{(1)}\left(x, x^{\prime}\right)=\left\langle E_{\mathrm{i}}^{*}(x) E_{\mathrm{i}}\left(x^{\prime}\right)\right\rangle$, the correlation propagator $H\left(x_{1}, x_{2} ; x, x^{\prime}\right)=\left\langle h_{\xi}^{*}\left(x_{1}, x\right) h_{\xi}\left(x_{2}, x^{\prime}\right)\right\rangle_{\xi}$ results from averaging over $\xi$, and $G_{\mathrm{o}}^{(1)}\left(x_{1}, x_{2}\right)=\left\langle\left\langle E_{\mathrm{o}}^{*}\left(x_{1}\right) E_{\mathrm{o}}\left(x_{2}\right)\right\rangle\right.$, where the double brackets indicate averaging over input field fluctuations and system disorder, which are assumed to be statistically independent. It is straightforward to generalize this formulation to twodimensional (2D) field distributions and spatially discretized systems such as coupled waveguide arrays.

We adopt a specific disordered linear systems ansatz for which the propagator $H$ factors in the form

$$
H\left(x_{1}, x_{2} ; x, x^{\prime}\right)=h_{\mathrm{a}}^{*}\left(x_{1}-x\right) h_{\mathrm{a}}\left(x_{2}-x^{\prime}\right) g_{\mathrm{a}}\left(x-x^{\prime}\right) .
$$

Note that the underlying realizations $h_{\xi}$ need not be shift invariant for the average impulse response $h_{a}(x)$ to be so; only the statistical properties of the system disorder are spatially stationary. Writing $G_{\mathrm{i}}^{(1)}\left(x, x^{\prime}\right)=\sqrt{I_{\mathrm{i}}(x) I_{\mathrm{i}}\left(x^{\prime}\right)} g_{\mathrm{i}}\left(x-x^{\prime}\right)$ in terms of the input intensity $I_{\mathrm{i}}(x)$ and the mutual degree of coherence $g_{\mathrm{i}}(x)$ [16], the imaging equation (1) becomes identical to that for a partially coherent system with effective impulse response function $h_{\mathrm{a}}(x)$ and mutual coherence function equal to the product $g(x)=g_{\mathrm{a}}(x) g_{\mathrm{i}}(x)$. In this case, the function $g_{\mathrm{a}}(x)$, which represents correlation in the disordered system, has the effect of modifying the degree of coherence of the input light (reducing its effective area of coherence).

Anderson localization occurs if the system disorder is such that a certain limit on $h_{\mathrm{a}}$ and $g_{\mathrm{a}}$ is attained. In the limit $h_{\mathrm{a}}(x) \rightarrow$ $\delta(x)$, Eq. (2) yields $G_{\mathrm{o}}^{(1)}\left(x_{1}, x_{2}\right) \rightarrow G_{\mathrm{i}}^{(1)}\left(x_{1}, x_{2}\right) g\left(x_{1}-x_{2}\right)$ and $I_{\mathrm{o}}\left(x_{1}\right) \rightarrow I_{\mathrm{i}}\left(x_{1}\right)$; that is, the point spread function of the system is a $\delta$ function. Regardless of $g_{\mathrm{a}}(x)$ and $g_{\mathrm{i}}(x)$, this is a perfect imaging system for intensity where a point input excitation leads to a highly localized output distribution. Furthermore, if the disorder is such that $g_{\mathrm{a}}(x) \rightarrow \delta(x)$, then $G_{\mathrm{o}}^{(1)}\left(x_{1}, x_{2}\right) \rightarrow$ $I_{\mathrm{i}}\left(x_{1}\right) \delta\left(x_{1}-x_{2}\right)$ and the output coherence function is that of 
an incoherent field. In this case, the disordered system washes out any partial coherence of the input field and suppresses any gain of coherence that usually results from propagation. The coherence function narrows or "localizes" in the $x_{1}=x_{2}$ direction in correlation space. We use this stronger limit, both $h_{\mathrm{a}}(x) \rightarrow \delta(x)$ and $g_{\mathrm{a}}(x) \rightarrow \delta(x)$, to represent the optical analog of AL, which then corresponds to a narrowing of the average impulse response function and a reduction in the coherence length.

The need for using point excitations to demonstrate $\mathrm{AL}$ is now clear. For a point excitation, say at $x=0, G_{\mathrm{o}}^{(1)}\left(x_{1}, x_{2}\right)=$ $h_{\mathrm{a}}^{*}\left(x_{1}\right) h_{\mathrm{a}}\left(x_{2}\right)$ and $I_{\mathrm{o}}(x)=\left|h_{\mathrm{a}}(x)\right|^{2}$, so that observing $\mathrm{AL}$ is tantamount to measuring $\left|h_{\mathrm{a}}(x)\right|^{2}$. An extended source, on the other hand, masks this outcome. For an extended input distribution with $g(x)=\delta(x)$, whether an incoherent input or a disordered system with $g_{\mathrm{a}}(x)=\delta(x)$, we have $I_{\mathrm{o}}\left(x_{1}\right)=$ $\int d x\left|h_{\mathrm{a}}\left(x_{1}-x\right)\right|^{2} I_{\mathrm{i}}(x)$, so that localization is masked by the convolution operation.

The statistical averaging needed for observing AL may be achieved by either exciting a fixed input point of multiple disordered-system realizations [6] or scanning input points of the same disordered system [4]. We have shown that these two approaches are equivalent [17]. Here we demonstrate that the parallelism enabled by quantum entanglement carries out the statistical averaging automatically in a manner similar to the latter strategy.

As a concrete realization of a disordered system, consider a one-dimensional (1D) array of coupled waveguides where the spatially discretized field amplitudes $E_{j}(z)$ in the $j$ th waveguide evolves according to $-i \frac{d E_{j}}{d z}=\beta_{n} E_{j}+\kappa\left(E_{j-1}+\right.$ $E_{j+1}$ ) [2]. Here $\kappa$ is the coupling coefficient between adjacent waveguides and the $\beta_{j}$ 's are random propagation constants chosen from a uniform probability distribution with mean $\beta_{o}$ and width $\Delta$. This model is the so-called diagonal disorder model. An alternative model, the so-called offdiagonal disorder, consists of a lattice of waveguides having random propagation constants and fixed coupling between the waveguides. While we use diagonal disorder throughout the paper, our conclusions are equally valid for the off-diagonal disorder model.

The input field $\mathbf{E}_{\mathrm{i}}=\left\{E_{j}(0)\right\}$ is related to the output field $\mathbf{E}_{\mathrm{o}}=\left\{E_{j}(z)\right\}$ through $\frac{d \mathbf{E}_{\mathrm{o}}}{d z}=i \hat{\mathbf{C}} \mathbf{E}_{\mathrm{i}}$, so that $\mathbf{E}_{\mathrm{o}}=\hat{\mathbf{h}}(z) \mathbf{E}_{\mathrm{i}}$, where the transfer matrix $\hat{\mathbf{h}}(z)=e^{i \hat{\mathbf{C}} z}$, and the propagator $\hat{\mathbf{H}}$ [Eq. (2)] results from the correlation of $\hat{\mathbf{h}}$. In Fig. 1(b) we plot the averaged output intensity distribution from arrays with different levels of disorder $\Delta$ ranging from an extended state in a periodic array $(\Delta=0)$ to an AL state in a disordered one. We use this discrete disordered-system model in our subsequent calculations of entangled-photon propagation.

We now proceed to examine the propagation of a general two-photon state with density operator

$$
\hat{\rho}=\iiint \int d x d x^{\prime} d x^{\prime \prime} d x^{\prime \prime \prime} \rho_{\mathrm{i}}\left(x, x^{\prime} ; x^{\prime \prime}, x^{\prime \prime \prime}\right)\left|1_{x}, 1_{x^{\prime}}\right\rangle\left\langle 1_{x^{\prime \prime}}, 1_{x^{\prime \prime \prime}}\right|
$$

in a disordered system in the AL limit [Fig. 1(c)] by evaluating the coincidence rate (intensity correlation) $G^{(2)}\left(x, x^{\prime}\right)=$ $\rho\left(x, x^{\prime} ; x, x^{\prime}\right)[18]$. We first consider the special case of a pure
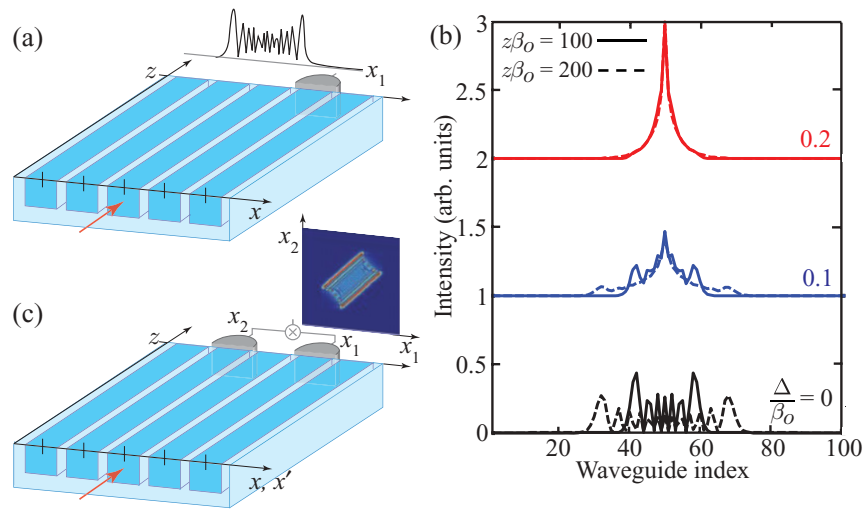

FIG. 1. (Color online) (a) Schematic of the system configuration with a coupled-waveguide array. (b) Intensity distribution at the output of arrays with different degrees of disorder $\frac{\Delta}{\beta_{o}}$ averaged over multiple realizations for two different lengths $z \beta_{o}$. The distributions are displaced vertically for clarity. (c) Schematic of a pair of photons propagating through a disordered system and the coincidence rate $G_{\mathrm{o}}^{(2)}\left(x_{1}, x_{2}\right)$ measured at the output.

two-photon state $|\Psi\rangle=\iint d x d x^{\prime} \psi\left(x, x^{\prime}\right)\left|1_{x}, 1_{x^{\prime}}\right\rangle$ representing entangled pairs correlated in their positions $\psi\left(x, x^{\prime}\right)=$ $\varphi(x) \delta\left(x-x^{\prime}\right)$. This idealized state may be generated in type I collinear degenerate spontaneous parametric down-conversion (SPDC) [19] in the thin-crystal narrowband limit $[11,15]$. The degree of entanglement of such a state depends on the width $w$ of $\varphi(x)$ (larger $w$ corresponds to stronger entanglement) [20]. The input coincidence rate $G_{i}^{(2)}\left(x_{1}, x_{2}\right)=\left|\varphi\left(x_{1}\right)\right|^{2} \delta\left(x_{1}-x_{2}\right)$ is diagonal along $x_{1}=x_{2}$ as a result of the photon-pair spatial correlation, and the output coincidence rate is

$$
G_{\mathrm{o}}^{(2)}\left(x_{1}, x_{2}\right)=\iint d x d x^{\prime} \varphi(x) \varphi^{*}\left(x^{\prime}\right) H_{+}^{(2)}\left(x_{1}, x_{2} ; x, x^{\prime}\right),
$$

where the propagator $H_{+}^{(2)}\left(x_{1}, x_{2} ; x, x^{\prime}\right)$ is given by

$$
\left\langle h_{\xi}\left(x_{1}, x\right) h_{\xi}^{*}\left(x_{1}, x^{\prime}\right) h_{\xi}\left(x_{2}, x\right) h_{\xi}^{*}\left(x_{2}, x^{\prime}\right)\right\rangle_{\xi},
$$

so that the coincidence rate in effect corresponds to double traversal through the system. We introduce two output marginal functions extracted from $G_{\mathrm{o}}^{(2)}\left(x_{1}, x_{2}\right)$ : (1) the singles rate $S_{\mathrm{o}}\left(x_{1}\right)=\int d x_{2} G_{\mathrm{o}}^{(2)}\left(x_{1}, x_{2}\right)$, resulting from integrating over $x_{2}$ and which corresponds to single-photon detection at the output, and (2) the diagonal marginals $S_{\mathrm{o}}^{+}\left(x_{1}\right)=$ $\int d x_{2} G_{\mathrm{o}}^{(2)}\left(x_{1}+x_{2}, x_{2}\right)$, resulting from integration along the $x_{1}=x_{2}$ direction.

In order to understand the consequences of Eq. (4), we first consider a periodic array, $\Delta=0$. In Fig. 2 we show the evolution of $G_{\mathrm{o}}^{(2)}\left(x_{1}, x_{2}\right)$ for various values of $z$. The output singles $S_{\mathrm{o}}\left(x_{1}\right)$ reflects the convolution of the input singles $S_{\mathrm{i}}\left(x_{1}\right)=\left|\varphi\left(x_{1}\right)\right|^{2}$ with the system point spread function. On the other hand, the diagonal marginal $S_{\mathrm{o}}^{+}\left(x_{1}\right)$ has the same distribution as that resulting from a point excitation at the input to a system of twice the physical length [Fig. 1(b)]. Note that this holds only for the entangled photon case as we see below when discussing the effect of partial entanglement.

A similar trend is observed in the disordered case $\left(\frac{\Delta}{\beta_{o}}=\right.$ 0.2 ). When input point excitations of classical light are coupled to this array, AL is observed [Fig. 1(b)]. When the entangled two-photon state propagates in this array, $G_{\mathrm{o}}^{(2)}\left(x_{1}, x_{2}\right)$ remains 

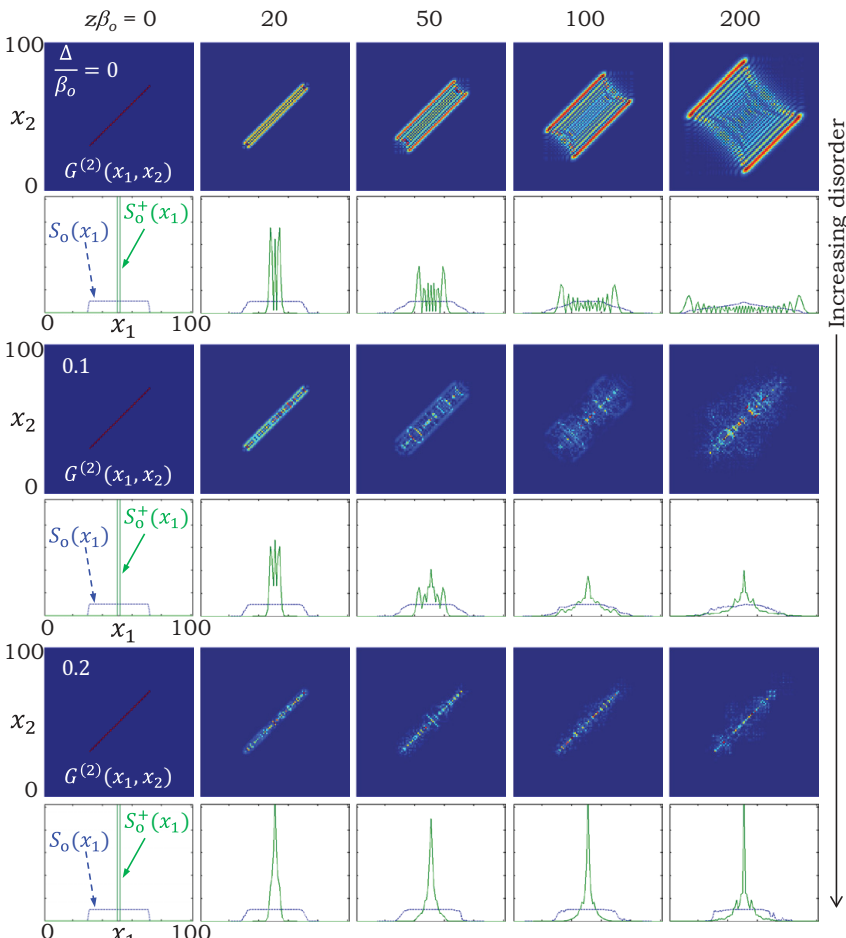

Increasing propagation distance

FIG. 2. (Color online) Output coincidence rate $G_{\mathrm{o}}^{(2)}\left(x_{1}, x_{2}\right)$ (2D color plots, $101 \times 101$ waveguides), single marginals $S_{\mathrm{o}}\left(x_{1}\right)(1 \mathrm{D}$ plots, dashed blue), and diagonal marginals $S_{\mathrm{o}}^{+}\left(x_{1}\right)$ (1D plots, solid green) for various propagation distances of entangled photons in $1 \mathrm{D}$ coupled waveguide arrays (each containing 101 waveguides with $\kappa=0.05$ ) with different degrees of disorder. The columns correspond to propagation distances $z \beta_{o}=0,20,50,100$, and 200. The first pair of rows corresponds to a periodic array $\Delta=0$, and the second and third pairs correspond to $\frac{\Delta}{\beta_{0}}=0.1$ and 0.2 , respectively; 41 waveguides are excited with equal amplitudes. The marginals $S_{0}\left(x_{1}\right)$ and $S_{\mathrm{o}}^{+}\left(x_{1}\right)$ in all panels are normalized to the same value for comparison; $G_{\mathrm{o}}^{(2)}\left(x_{1}, x_{2}\right)$ in each panel is normalized separately for clarity.

diagonal along $x_{1}=x_{2}$. Since $S_{\mathrm{o}}\left(x_{1}\right)$ is spatially extended, neither photon separately demonstrates AL. On the other hand, the narrow distribution of $S_{\mathrm{o}}^{+}\left(x_{1}\right)$ centered on $x_{1}=0$ signifies that the separation between the two photons is always small, and in the AL limit the two photons always emerge together from the same waveguide into which they entered. In other words, neither photon localizes, but the pair colocalizes. We term this phenomenon Anderson colocalization (AcL).

Furthermore, entangled photons colocalize faster in twophoton correlation space than a classical field localizes when traversing the same medium. This is clearly seen when we compare the output intensity $I_{0}\left(x_{1}\right)$ for classical input point excitation [Fig. 1(b)] and $S_{\mathrm{o}}^{+}\left(x_{1}\right)$ for entangled pairs (Fig. 2). We find that $S_{\mathrm{o}}^{+}\left(x_{1}\right)$ corresponding to a propagation length $z$ is identical to $I_{0}\left(x_{1}\right)$ for length $2 z$, as was anticipated from Eq. (4).

The entangled state is a useful simplification, but it is also important to examine how partial entanglement affects AcL. We consider here the effect of the decrease in the entangledphoton source width $w$. As $w$ decreases, the strength of the photon correlations decreases, and the two photons become

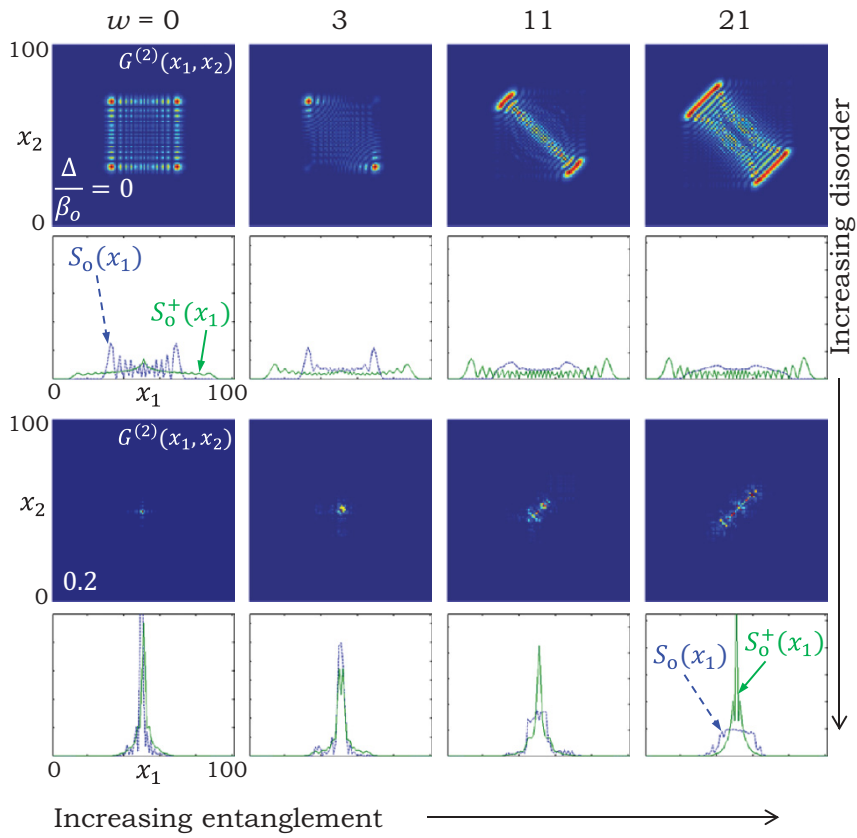

FIG. 3. (Color online) Effect of entangled-photon source size, and hence degree of entanglement, on the two-photon coincidence rate. The first two rows depict $G_{\mathrm{o}}^{(2)}\left(x_{1}, x_{2}\right)$ and both the single marginals $S_{\mathrm{o}}\left(x_{1}\right)$ (dashed blue) and diagonal marginals $S_{\mathrm{o}}^{+}\left(x_{1}\right)$ (solid green) for a periodic array $(\Delta=0)$ for various values of the width $w$. The last two rows correspond to a disordered array $\left(\frac{\Delta}{\beta_{0}}=0.2\right)$. Note the evolution from $\mathrm{AL}$ when $w=1$ (blue curve on the left) to AcL for larger width (green curve on the right). Normalization scheme and waveguide index range are similar to Fig. 2 and $z \beta_{o}=200$.

uncorrelated when the source is reduced to a point [20]. In Fig. 3 we depict $G_{0}^{(2)}\left(x_{1}, x_{2}\right)$ for periodic $(\Delta=0)$ and disordered $\left(\frac{\Delta}{\beta_{o}}=0.2\right)$ arrays while increasing $w$ from 1 to 21 in units of interwaveguide separation. When $w=1$, the two photons are no longer entangled and they enter together one waveguide. Consequently $G_{\mathrm{o}}^{(2)}\left(x_{1}, x_{2}\right)$ factorizes into a product since the two photons are uncorrelated. Here $S_{\mathrm{o}}\left(x_{1}\right)$ is identical to $I_{0}\left(x_{1}\right)$ for a point input excitation at $x=0$. This behavior was previously noted in Ref. [8]. In this case $S_{\mathrm{o}}^{+}\left(x_{1}\right)$ does not carry any information about the system. As we increase $w$, the source becomes entangled and $G_{\mathrm{o}}^{(2)}\left(x_{1}, x_{2}\right)$ no longer factorizes. In the periodic array, the two diagonal peaks are diminished and the two off-diagonal peaks are strengthened. $S_{\mathrm{o}}\left(x_{1}\right)$ no longer corresponds to the classical output and the discrete diffraction features are washed out. On the other hand, $S_{\mathrm{o}}^{+}\left(x_{1}\right)$ approaches $I_{\mathrm{o}}\left(x_{1}\right)$ for a point input excitation to a system with twice the length of the physical system. For the disordered array, $G_{\mathrm{o}}^{(2)}\left(x_{1}, x_{2}\right)$ factorizes when $w=1$ and both photons exhibit $A L$. In this case both $S_{\mathrm{o}}\left(x_{1}\right)$ and $S_{\mathrm{o}}^{+}\left(x_{1}\right)$ reflect the mapping of the localized input state to a localized output state. As $w$ increases, $S_{0}\left(x_{1}\right)$ broadens in step with $w$, while $S_{\mathrm{o}}^{+}\left(x_{1}\right)$ maintains the small width associated with AcL. Increasing entanglement thus results in a gradual evolution from AL to AcL.

We next consider the entangled two-photon state when the two photons are anticorrelated in position $\psi\left(x, x^{\prime}\right)=$ $\varphi(x) \delta\left(x+x^{\prime}\right)$, which may be produced after the two entangled photons with correlated positions traverse a Fourier-transform 
(a)
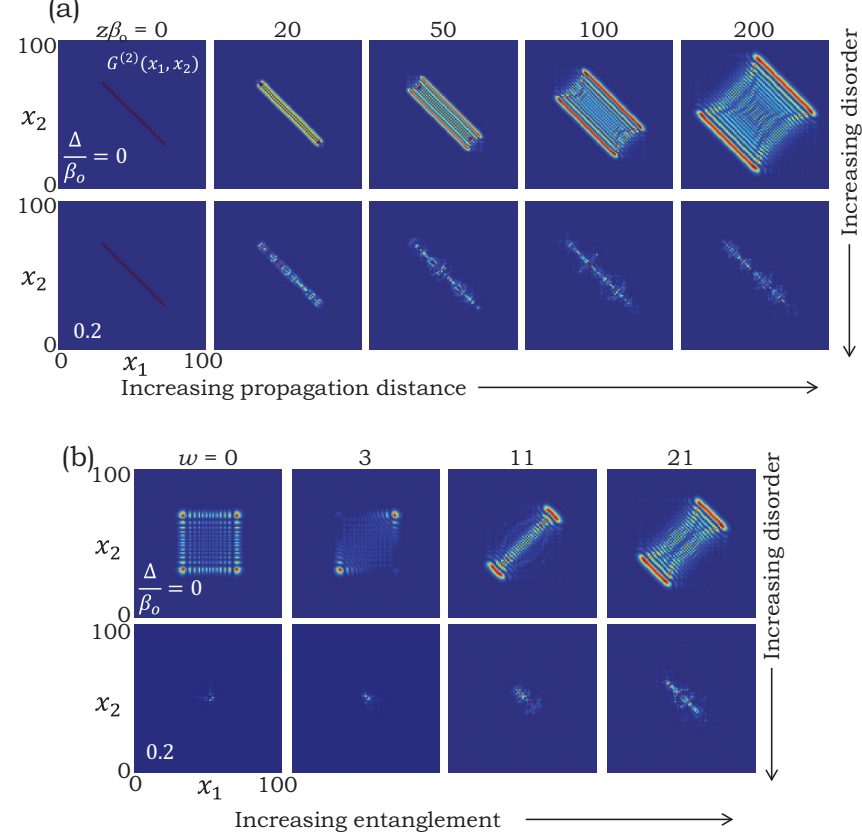

FIG. 4. (Color online) Anderson localization and colocalization of entangled photon pairs anticorrelated in position. (a) Propagation dynamics of entangled photon pairs in periodic ( $\Delta=0$, first row) and disordered $\left(\frac{\Delta}{\beta_{o}}=0.2\right.$, second row) arrays. (b) Effect of partial entanglement on AL and AcL; $z \beta_{o}=200$. Normalization scheme is similar to Fig. 2. Waveguide index range is similar to Figs. 2 and 3.

system [11,15]. The two photons in such a state are always on opposite sides of the origin at the input, and the output coincidence rate is

$$
G_{\mathrm{o}}^{(2)}\left(x_{1}, x_{2}\right)=\iint d x d x^{\prime} \varphi(x) \varphi^{*}\left(x^{\prime}\right) H_{-}^{(2)}\left(x_{1}, x_{2} ; x, x^{\prime}\right),
$$

where the propagator $H_{-}^{(2)}\left(x_{1}, x_{2} ; x, x^{\prime}\right)$ is given by

$$
\left\langle h_{\xi}\left(x_{1}, x\right) h_{\xi}^{*}\left(x_{1}, x^{\prime}\right) h_{\xi}\left(x_{2},-x\right) h_{\xi}^{*}\left(x_{2},-x^{\prime}\right)\right\rangle_{\xi} .
$$

We find that the results are identical to the correlated case except that the $x_{1}=x_{2}$ and $x_{1}=-x_{2}$ diagonals in $G_{\mathrm{o}}^{(2)}\left(x_{1}, x_{2}\right)$ are exchanged, as shown in Fig. 4. We observe AcL here in the narrow width of $G_{\mathrm{o}}^{(2)}\left(x_{1}, x_{2}\right)$ along the $x_{1}=-x_{2}$ direction. Hence, the antidiagonal marginal $S_{\mathrm{o}}^{-}\left(x_{1}\right)=\int d x_{2} G_{\mathrm{o}}^{(2)}\left(x_{1}-\right.$ $\left.x_{2}, x_{2}\right)$ exhibits AcL here. In this case, the separation between the two photons remains invariant, and the two photons always emerge symmetrically from opposite sides of the array.

An important and general observation emerges from the results above: For entangled photons, whether correlated (Fig. 2) or anticorrelated (Fig. 4), the coincidence rates at the output $G_{\mathrm{o}}^{(2)}\left(x_{1}, x_{2}\right)=\rho_{\mathrm{o}}\left(x_{1}, x_{2} ; x_{1}, x_{2}\right)$ and input $G_{\mathrm{i}}^{(2)}\left(x_{1}, x_{2}\right)=\rho_{\mathrm{i}}\left(x_{1}, x_{2} ; x_{1}, x_{2}\right)$ are, surprisingly, the same, since $\rho_{\mathrm{o}}\left(x_{1}, x_{2} ; x_{1}^{\prime}, x_{2}^{\prime}\right) \rightarrow \rho_{\mathrm{i}}\left(x_{1}, x_{2} ; x_{1}, x_{2}\right) \delta\left(x_{1}-\right.$ $\left.x_{1}^{\prime}\right) \delta\left(x_{2}-x_{2}^{\prime}\right)$ in the AL limit. This result applies in fact to all two-photon states in the AL limit. It is not the case, though, that the output and input states remain necessarily the same; only the coincidence rates do. An AL system thermalizes the two-photon state and $\rho_{\mathrm{o}}$ becomes diagonal regardless of the input state. There is, though, a family of two-photon states that remain unchanged after passage through a disordered $\mathrm{AL}$ medium. We call these states two-photon AL eigenstates, and they take the form

$$
\rho_{\mathrm{i}}\left(x, x^{\prime} ; x^{\prime \prime}, x^{\prime \prime \prime}\right)=\rho\left(x, x^{\prime} ; x, x^{\prime}\right) \delta\left(x-x^{\prime \prime}\right) \delta\left(x^{\prime}-x^{\prime \prime \prime}\right) .
$$

Examples of such eigenstates include (1) separable mixed states $\rho_{\mathrm{i}}\left(x, x^{\prime} ; x^{\prime \prime}, x^{\prime \prime \prime}\right)=\rho_{1}(x) \delta\left(x-x^{\prime \prime}\right) \rho_{2}\left(x^{\prime}\right) \delta\left(x^{\prime}-\right.$ $\left.x^{\prime \prime \prime}\right)$, where the two photons are independent and mixed, and (2) classically correlated states $\rho_{\mathrm{i}}\left(x, x^{\prime} ; x^{\prime \prime}, x^{\prime \prime \prime}\right)=\rho(x) \delta(x-$ $\left.x^{\prime}\right) \delta\left(x-x^{\prime \prime}\right) \delta\left(x^{\prime}-x^{\prime \prime \prime}\right)$, where the two photons are correlated in location, as in the entangled-photon case, but lack phase correlations. We thus uncover a central result of this Rapid Communication: AL does not diminish two-photon spatial correlations, but it does destroy phase correlations. Separable two-photon states remain separable; correlated two-photon states remain correlated.

In conclusion, we have presented a formalism to describe the propagation of classical and two-photon light in disordered systems. We uncovered a phenomenon associated with the propagation of entangled-photon pairs in such systems, which we call Anderson colocalization: While neither photon is localized, the two-photon separation in coincidence space $i s$. The increase in entanglement is accompanied by a gradual evolution from AL to AcL. Our results are intimately connected with a specific ansatz for a disordered system model inspired by the waveguide arrays used heretofore in AL demonstrations with classical light. This work should stimulate further investigations of higher-order correlation functions of classical and quantum states of light propagating in disordered media using our model and other variants.
[1] P. W. Anderson, Phys. Rev. 109, 1492 (1958).

[2] D. N. Christodoulides, F. Lederer, and Y. Silberberg, Nature (London) 424, 817 (2003).

[3] H. De Raedt, A. Lagendijk, and P. de Vries, Phys. Rev. Lett. 62, 47 (1989); T. Schwartz, G. B. S. Fishman, and M. Segev, Nature (London) 446, 52 (2007).

[4] Y. Lahini et al., Phys. Rev. Lett. 100, 013906 (2008).

[5] Y. Lahini et al., Phys. Rev. Lett. 103, 013901 (2009).

[6] A. Szameit et al., Opt. Lett. 35, 1172 (2010).

[7] D. Blömer et al., Opt. Express 14, 2151 (2006).
[8] Y. Bromberg, Y. Lahini, R. Morandotti, and Y. Silberberg, Phys. Rev. Lett. 102, 253904 (2009)

[9] G. D. Marshall et al., Opt. Express 17, 12546 (2009); Y. Lahini, Y. Bromberg, D. N. Christodoulides, and Y. Silberberg, Phys. Rev. Lett. 105, 163905 (2010); A. Peruzzo et al., Science 329, 1500 (2010); J. O. Owens et al., arXiv:1103.0604.

[10] M. A. Nielsen and I. L. Chuang, Quantum Computation and Quantum Information (Cambridge University Press, Cambridge, 2000). 
[11] B. E. A. Saleh, A. F. Abouraddy, A. V. Sergienko, and M. C. Teich, Phys. Rev. A 62, 043816 (2000).

[12] C. W. J. Beenakker, J. W. F. Venderbos, and M. P. van Exter, Phys. Rev. Lett. 102, 193601 (2009); W. H. Peeters, J. J. D. Moerman, and M. P. van Exter, ibid. 104, 173601 (2010); J. R. Ott, N. A. Mortensen, and P. Lodahl, ibid. 105, 090501 (2010).

[13] N. Cherroret and A. Buchleitner, Phys. Rev. A 83, 033827 (2011).

[14] C. Thompson, G. Vemuri, and G. S. Agarwal, Phys. Rev. A 82, 053805 (2010).
[15] A. F. Abouraddy et al., J. Opt. Soc. Am. B 19, 656 (2001).

[16] L. Mandel and E. Wolf, Optical Coherence and Quantum Optics (Cambridge University Press, New York, 1995).

[17] L. Martin et al., Opt. Express 19, 13636 (2011).

[18] R. J. Glauber, Phys. Rev. 130, 2529 (1963).

[19] S. E. Harris, M. K. Oshman, and R. L. Byer, Phys. Rev. Lett. 18, 732 (1967).

[20] A. F. Abouraddy, M. B. Nasr, B. E. A. Saleh, A. V. Sergienko, and M. C. Teich, Phys. Rev. A 63, 063803 (2001); C. K. Law and J. H. Eberly, Phys. Rev. Lett. 92, 127903 (2004). 